\title{
Pesquisa Qualitativa: Grupo Focal e Intervenções Psicológicas com Idosos
}

Psychologically Focused Group Intervention with the Elderly: a Qualitative Research

Investigación Cualitativa: Grupo Focal e Intervenciones Psicológicas con Ancianos

Ana Lúcia Gatti, Carla Witter, Claúdia Aranha Gil \& Sueli dos Santos Vitorino

Universidade São Judas Tadeu

http://dx.doi.org/10.1590/1982-3703002382013 
Resumo: A realização de pesquisas qualitativas é bastante frequente nas ciências sociais, entretanto pouco comum para investigar o processo de envelhecimento no Brasil, embora haja o esforço dos pesquisadores na utilização de métodos tais como: grupo focal, grupos operativos e oficinas psicoterapêuticas. O objetivo deste ensaio teórico foi conceituar e discutir criticamente a utilização dessa modalidade de investigação na produção de conhecimento científico sobre idosos. Com base na literatura e na reflexão dos próprios autores, discutem-se questões pertinentes aos métodos qualitativos referidos, especialmente na condução de estudos e intervenções fundamentados no método clínico. Para embasar e aprofundar o conhecimento sobre a utilização da técnica de grupo focal com idosos em pesquisas, foi feito levantamento da produção científica na SciELO, LILACS e PsycINFO sobre o tema, sendo discutidos os 18 artigos capturados. Para exemplificar o uso de intervenções psicológicas com idosos nas pesquisas qualitativas, são descritos o aporte teórico, os métodos e os resultados gerais de uma intervenção psicológica e de uma oficina terapêutica. Concluiu-se que as pesquisas qualitativas agregam conhecimento importante sobre os idosos e seu processo de envelhecimento, sendo destacado o benefício das intervenções e a possibilidade de aprofundar o assunto com a utilização de métodos qualitativos.

Palavras-chave: Metodologia. Psicoterapia. Gerontologia. Envelhecimento.

Abstract: Conducting qualitative research is quite common in social sciences; however, this type of research is not very frequently used in Brazil to investigate the aging process despite the efforts of researchers in using methods such as focus groups, operational groups and psychotherapeutic workshops. The aim of this theoretical paper was to conceptualize and critically discuss the use of qualitative research in the production of scientific knowledge about the elderly. Based on the literature and on the authors' own reflections, this study discusses matters relevant to the previously mentioned qualitative methods, especially regarding the conduction of studies and interventions based on the clinical method. To support and deepen the knowledge on the use of the focus group technique in research with the elderly, a search for the scientific production on this theme was performed at SciELO, LILACS, PsycINFO databases. Eighteen articles were selected for discussion. To exemplify the use of psychological interventions with older adults in qualitative research, the theoretical framework, methods and overall results of a psychological intervention and therapeutic workshop are described. We conclude that qualitative studies add important knowledge about the elderly and the aging process, highlighting the benefit of interventions and the possibility of developing a more comprehensive knowledge on the subject through the use of qualitative methods.

Keywords: Methodology. Psychotherapy.Gerontology.Aging.

Resumen: La realización de investigaciones cualitativas es muy frecuente en las ciencias sociales, pero poco utilizada a la hora de investigar el proceso de envejecimiento en Brasil, aunque los investigadores se esfuerzan en el uso de estos métodos: grupo focal, grupos operativos y talleres psicoterapéuticos. El objetivo de este trabajo teórico fue conceptualizar y discutir críticamente el uso de este tipo de investigación en la producción de conocimiento científico sobre los ancianos. Con base en la literatura y en la propia reflexión de los autores, se discuten cuestiones relevantes acerca de los métodos cualitativos citados, sobre todo en la realización de estudios e intervenciones apoyados en el método clínico. Para apoyar y profundizar el conocimiento sobre el uso de la técnica de grupo focal con ancianos en investigaciones, se hizo una averiguación de producción científica en SciELO, LILACS, PsycINFO sobre el tema, siendo debatidos los 18 artículos capturados. Para ejemplificar el uso de intervenciones psicológicas con ancianos en las investigaciones cualitativas, se describen el marco teórico, los métodos y los resultados generales de una intervención psicológica y de un taller terapéutico. Se concluyó que los estudios cualitativos agregan conocimiento importante sobre los ancianos y su proceso de envejecimiento, destacándose el beneficio de las intervenciones y la posibilidad de profundizar en el tema con el uso de métodos cualitativos.

Palabras-clave: Metodología. Psicoterapia. Gerontología. Envejecimiento. 
A conceituação de grupo é bastante diversa quando tomada em contextos e disciplinas diferentes, como por exemplo a administração, a Antropologia, a Sociologia e a Psicologia. Entretanto, importa extrair, assim como o faz Osório (2003), uma definição operacional, de modo a permitir um breve esboço da história das teorias e técnicas referentes aos trabalhos grupais nas várias áreas do conhecimento.

Um grupo humano é um conjunto de pessoas, com objetivos compartilhados e uma ação interativa (Osório, 2003). São indivíduos que se reconhecem em sua singularidade e estão submetidos às diversas leis que se impõem na situação grupal, as quais começaram a ganhar maior estudo ao final da década de 1940, com os trabalhos de Bion, mas que tiveram suas raízes nas obras de Freud, como Totem e tabu, de 1913, e com destaque especial para Psicologia das massas e análise do ego, de 1921 (Kernberg, 2000).

Se, quanto à atividade prática, Slavson foi o pioneiro quanto aos trabalhos com grupos terapêuticos nos Estados Unidos, o que ocorreu na década de 1930, as décadas de 50 e 60 do século passado evidenciaram um grande desenvolvimento sobre o tema, com a incorporação de conhecimentos tanto teóricos quanto técnicos advindos da Sociologia. Destacam-se no período as contribuições de Kurt Lewin, Enrique Pichòn-Rivere e José Bleger, o que redundou em uma ampliação também da aplicação das técnicas grupais para diversas finalidades e diversos contextos, os quais não mais se restringiam às terapias, nem à área da saúde, como é a situação do emprego da técnica grupal do modo que ocorre nos grupos focais (Osório, 2003).

A técnica do grupo focal tem sido indicada como um instrumento de pesquisa qualitativa que permite levantar e compreender, do grupo de informantes, suas opiniões, relevâncias e valores, a partir de uma discussão que ocorre em reuniões com um pequeno número de participantes e, geralmente, com um animador, o qual busca focalizar e aprofundar as discussões por meio de suas intervenções (Minayo, 2006). Normalmente os grupos focais são compostos de seis a dez participantes com características comuns que se reúnem em encontros semanais de uma hora e meia, nos quais é discutido um tema específico ou focal, sendo as discussões moderadas pelo animador, que pode também atuar como pesquisador (Carlini-Cotrim, 1996).

Pela possibilidade que oferece de coleta de dados com riqueza, flexibilidade e espontaneidade (Oliveira \& Freitas, 1998), os grupos focais têm sido utilizados em pesquisas nas áreas sociais e no campo da saúde, em que, segundo Backes, Colomé, Erdmann \& Lunardi (2011, p. 438), podem "instigar novos saberes, [...] ressignificar posturas profissionais e [...] aproximar a pesquisa dos cenários de prática e vice-versa". Por exemplo, o trabalho de Coutinho, Diogo, Joaquim \& Borges (2011) apresenta o uso do grupo focal para compreender os sentidos atribuídos pelos servidores públicos de uma universidade sobre as transformações ocorridas nos setores produtivos nacionais. Essa técnica tem sido utilizada, inclusive, como instrumento de avaliação de treinamento de profissionais da saúde (Soares, Reale \& Brites, 2000).

O campo da saúde e o processo de envelhecimento têm estado cada vez mais imbricados em nossa sociedade contemporânea, dado o aumento da longevidade e as requisições de cuidado que a população idosa traz, dadas as diversas perdas potenciais nos aspectos físicos, psicológicos e sociais que vivencia, a exemplo de reduções de algumas capacidades físicas (Salmaso et al., 2014), de perdas de pessoas significativas (Rubio, Wanderley \& Ventura, 2011) e de papéis (Bruns \& Abreu, 1997; Debert, 1999; Bosi, 2003). Assim, como propõem Backes et al. (2011), a técnica de grupo focal seria mais uma possibilidade de pesquisar os idosos e intervir no processo de envelhecimento, na medida em que possibilita a geração de conhecimento a partir da discussão de temáticas específicas, como vida e morte ou bem-estar e qualidade de vida, de acordo com a compreensão do idoso sobre o tema focal.

A se considerar que as pessoas que envelhecem têm o direito de expressar suas opiniões e valores no âmbito da sociedade em que estejam inseridas, é de se esperar que os grupos focais venham sendo utilizados com frequência na sondagem dos assuntos que Ihes dizem respeito, isto é, pesquisas tanto 
na área das ciências sociais como na saúde. Com o intuito de verificar o que foi produzido nos anos recentes por meio dos grupos focais com a população idosa, realizou-se um levantamento em bases que congregam trabalhos científicos - LILACS, SciELO e PsycINFO, o que caracteriza uma pesquisa de produção científica, a qual permite verificar o estado da arte sobre o tema (Witter, 2010; Witter \& Buriti, 2011).

Há diversos trabalhos de metaciência que investigam um determinado tema de relevância para a compreensão do processo de envelhecimento. Por exemplo, os trabalhos de Dawalibi, Anacleto, Witter, Goulart \& Aquino (2013) e de Witter, Buriti, Silva, Nogueira \& Gama(2013) analisaram, respectivamente, a produção científica sobre envelhecimento e qualidade de vida na SciELO e sobre dança na Biblioteca Virtual de Saúde (BVS). A pesquisa de Dawalibi et al. (2013) encontrou 69 artigos sobre a temática da qualidade de vida, sendo o predomínio das publicações nas áreas de Psicologia $(26,1 \%)$ em Ciências Humanas e de Medicina $(21,4 \%)$ em Ciências da Saúde. Já o estudo de Witter et al. (2013) capturou 68 artigos sobre dança e envelhecimento, sendo que a maioria dos trabalhos era de pesquisas de campo com idosos (82,35\%), sendo $57,36 \%$ de estudos descritivos. Em ambos os trabalhos, os autores analisaram dados metacientíficos relacionados a questões de autoria, de metodologia (delineamento das pesquisas), temática, entre outros aspectos.
Em particular, para a análise do conhecimento científico publicado sobre grupo focal nas bases do PsycINFO, LILACS e SciELO, definiu-se verificar: a autoria, apresentando o nome dos autores e ano de publicação; o método, detalhando o número e o tipo de participantes ( $\mathrm{N}$ amostral) e denominação (idoso, família, adolescente, profissional de saúde etc); material utilizado para coleta de dados; tipo de procedimento de intervenção realizado, além do grupo focal; temática discutida no artigo e as principais conclusões do trabalho publicado. Para a captura dos artigos foram utilizadas as seguintes palavras-chave: idosos/elderly e envelhecimento/aging com a expressão grupo focal/focus group, sendo delimitado apenas o período de tempo para a busca no PsycINFO, de 2010 até 2012. Na LILACS, em que não houve restrição temporal para a busca, foram encontrados 15 trabalhos, na SciELO apenas um, já na PsycINFO obtiveram-se 231 na busca inicial. Entretanto, após a exclusão de teses, dissertações, livros e capítulos, e a análise do conteúdo, tem-se que, no total, 18 artigos trataram da técnica de grupo focal e a população idosa, sendo 14 na PsycINFO e quatro na LILACS.

Foi possível verificar que os idosos são o grupo exclusivamente consultado em 12 dos trabalhos, dado que em seis eles compõem a amostra juntamente com pessoas de outras idades ou pessoas em outras condições, como profissionais, familiares, estudantes etc. (Tabela 1). Houve uma grande variação no número de

Tabela 1. Artigos científicos sobre idosos e grupo focal nas bases de dados PsycINFO e LILACS

\begin{tabular}{|c|c|c|c|c|c|}
\hline Autor & $\begin{array}{c}\text { Participantes } \\
(\mathrm{N})\end{array}$ & Material & $\begin{array}{c}\text { Procedimento/ } \\
\text { Intervenção }\end{array}$ & Temática & Conclusões \\
\hline \multicolumn{6}{|c|}{ PsycINFO - American Psychology Association - busca de $2010-2012(\mathrm{~N}=231)$} \\
\hline $\begin{array}{l}\text { Moreau et al., } \\
2012\end{array}$ & $\begin{array}{l}\text { Idosos, usuários } \\
\text { de grupos de } \\
\text { apoio, estudantes } \\
\text { e habitantes rurais } \\
\text { (SI) }\end{array}$ & $\begin{array}{c}\text { Sem } \\
\text { informação } \\
\text { (SI) }\end{array}$ & Grupo focal & $\begin{array}{l}\text { Tomada de } \\
\text { decisão sobre } \\
\text { saúde }\end{array}$ & $\begin{array}{c}\text { Compartilhar a tomada de } \\
\text { decisão pode ser flexível } \\
\text { e integrado com outros } \\
\text { modelos paternalistas e } \\
\text { informativos }\end{array}$ \\
\hline $\begin{array}{l}\text { Bhagat, Laskar } \\
\text { e Sharma, } \\
2012\end{array}$ & $\begin{array}{l}\text { Adolescentes, } \\
\text { adultos e idosos } \\
\text { (42) }\end{array}$ & SI & Grupo focal & $\begin{array}{l}\text { Escolha do filho } \\
\text { por sexo em } \\
\text { favelas }\end{array}$ & $\begin{array}{l}\text { Há escolha do sexo } \\
\text { masculino e uso de métodos } \\
\text { de detecção }\end{array}$ \\
\hline
\end{tabular}




\begin{tabular}{|c|c|c|c|c|c|}
\hline Autor & $\begin{array}{c}\text { Participantes } \\
(\mathrm{N})\end{array}$ & Material & $\begin{array}{l}\text { Procedimento/ } \\
\text { Intervenção }\end{array}$ & Temática & Conclusões \\
\hline \multicolumn{6}{|c|}{ PsyclNFO - American Psychology Association - busca de $2010-2012(\mathrm{~N}=231)$} \\
\hline Conde, 2012 & $\operatorname{Idosos}(\mathrm{SI})$ & $\begin{array}{c}\text { Entrevista } \\
\text { semiestruturada }\end{array}$ & Grupo focal & Lazer & $\begin{array}{l}\text { Há distinção entre o lazer } \\
\text { oferecido na comunidade e } \\
\text { lazer para o idoso }\end{array}$ \\
\hline $\begin{array}{c}\text { MacKinlay e } \\
\text { Dundon, } 2012\end{array}$ & Idosos (SI) & $\mathrm{SI}$ & Grupo focal & $\begin{array}{l}\text { Bem-estar e } \\
\text { religiosidade }\end{array}$ & SI \\
\hline $\begin{array}{l}\text { Wu, Fassert e } \\
\text { Rigaud, } 2012\end{array}$ & Idosos $(\mathrm{SI})$ & $\mathrm{SI}$ & Grupo focal & $\begin{array}{l}\text { Desenhos de } \\
\text { robôs para } \\
\text { assistência de } \\
\text { idosos }\end{array}$ & $\begin{array}{c}\text { Aparência criticada, robôs } \\
\text { pequenos e criativos } \\
\text { elogiados; problemas éticos } \\
\text { e sociais }\end{array}$ \\
\hline $\begin{array}{c}\text { Zhan, Feng, } \\
\text { Chen \& Feng, } \\
2011\end{array}$ & $\operatorname{Idosos}(\mathrm{SI})$ & $\mathrm{SI}$ & $\begin{array}{l}\text { Grupo focal } \\
\text { e pesquisa de } \\
\text { levantamento }\end{array}$ & $\begin{array}{l}\text { Instituição } \\
\text { de longa } \\
\text { permanência } \\
\text { (ILP) e papel da } \\
\text { família }\end{array}$ & $\begin{array}{l}\text { Famílias chinesas: } \\
\text { dificuldades financeiras e } \\
\text { emocionais para deixar o } \\
\text { idoso numa ILP }\end{array}$ \\
\hline $\begin{array}{l}\text { Ellis, Mullan e } \\
\text { Worsley, } 2011\end{array}$ & $\begin{array}{l}\text { Idosos, + de } 65 \\
\text { anos }(28 \text { e } 226)\end{array}$ & SI & $\begin{array}{l}\text { Grupo focal } \\
\text { e pesquisa de } \\
\text { levantamento }\end{array}$ & $\begin{array}{c}\text { Comportamento } \\
\text { de acumulação, } \\
\text { empréstimo } \\
\text { ou partilha } \\
\text { de prescrição } \\
\text { médica }\end{array}$ & $\begin{array}{l}\text { Prevalência baixa do } \\
\text { comportamento; partilha } \\
\text { da prescrição e de } \\
\text { medicamentos para dor } \\
\text { forte são aceitáveis nessa } \\
\text { população }\end{array}$ \\
\hline $\begin{array}{l}\text { Walsh, Olson, } \\
\text { Ploeg, Lohfeld } \\
\text { \& MacMillan, } \\
2011\end{array}$ & Idosos $(\mathrm{SI})$ & $\mathrm{SI}$ & Grupo focal & $\begin{array}{c}\text { Abuso e } \\
\text { opressão; } \\
\text { violência contra } \\
\text { idosos }\end{array}$ & $\begin{array}{c}\text { Aumento da vulnerabilidade } \\
\text { para abusos está relacionada } \\
\text { à vivência de opressão }\end{array}$ \\
\hline $\begin{array}{l}\text { Cheng, Lo, } \\
\text { Chan \& Woo, } \\
2010\end{array}$ & Idosos (26) & $\begin{array}{l}\text { Questionário } \\
\text { de QV e Escala } \\
\text { de Depressão } \\
\text { Geriátrica }\end{array}$ & Grupo focal & $\begin{array}{c}\text { Terapia de luto } \\
\text { antecipatório } \\
\text { AGT - } \\
\text { anticipatory } \\
\text { brief therapy }\end{array}$ & $\begin{array}{c}\text { AGT é útil, viável e aceitável } \\
\text { para idosos que enfrentam } \\
\text { o fim da vida, tanto em } \\
\text { hospital e ILP }\end{array}$ \\
\hline $\begin{array}{l}\text { Fadda, Cortés, } \\
\text { Olivi \& } \\
\text { Tovar,2010 }\end{array}$ & Idosos (SI) & SI & $\begin{array}{l}\text { Grupo focal } \\
\text { e intervenção } \\
\text { etnográfica }\end{array}$ & Espaço urbano & $\begin{array}{c}\text { Topografia irregular e } \\
\text { patrimônio da cidade } \\
\text { influenciam a percepção do } \\
\text { espaço urbano, a criação de } \\
\text { redes sociais e o senso de } \\
\text { comunidade }\end{array}$ \\
\hline $\begin{array}{l}\text { Hutchings et al., } \\
2010\end{array}$ & $\begin{array}{l}\text { Idosos, família e } \\
\text { profissionais da } \\
\text { saúde }(12,11,16)\end{array}$ & $\begin{array}{c}\text { Entrevista } \\
\text { semiestruturada }\end{array}$ & Grupo focal & $\begin{array}{l}\text { Tratamento } \\
\text { com drogas; } \\
\text { Alzheimer }\end{array}$ & $\begin{array}{l}\text { Tratamentos influenciados: } \\
\text { percepções de } \\
\text { disponibilidade de } \\
\text { tratamento, eficácia, } \\
\text { capacidade de recursos e } \\
\text { benefícios }\end{array}$ \\
\hline $\begin{array}{l}\text { Frank et al., } \\
\quad 2010\end{array}$ & $\begin{array}{l}\text { Pacientes idosos, } \\
\text { médicos e } \\
\text { cuidadores (18, 9, } \\
22 \text { US e } 11 \text { UK) }\end{array}$ & $\begin{array}{c}\text { Escala de } \\
\text { dependência } \\
\text { e entrevista } \\
\text { qualitativa }\end{array}$ & Grupo focal & $\begin{array}{l}\text { Escala de } \\
\text { dependência, } \\
\text { Alzheimer }\end{array}$ & $\begin{array}{c}\text { Escala de dependência pode } \\
\text { ser valiosa para avaliar a } \\
\text { dependência em futuras } \\
\text { pesquisas de ensaios clínicos } \\
\text { de demência }\end{array}$ \\
\hline
\end{tabular}




\begin{tabular}{|c|c|c|c|c|c|}
\hline Autor & $\begin{array}{c}\text { Participantes } \\
(\mathrm{N})\end{array}$ & Material & $\begin{array}{c}\text { Procedimento/ } \\
\text { Intervenção }\end{array}$ & Temática & Conclusões \\
\hline \multicolumn{6}{|c|}{ PsyclNFO - American Psychology Association - busca de $2010-2012(\mathrm{~N}=231)$} \\
\hline $\begin{array}{l}\text { Iliffe et al., } \\
2010\end{array}$ & Idosos $(\mathrm{SI})$ & $\begin{array}{l}\text { Health Risk } \\
\text { Appraisal in } \\
\text { Older People } \\
\text { (HRAO) }\end{array}$ & Grupo focal & $\begin{array}{l}\text { Política pública } \\
\text { para o bem- } \\
\text { estar de idosos }\end{array}$ & $\begin{array}{l}\text { Identificou os principais } \\
\text { determinantes sociais da } \\
\text { saúde para idosos e para o } \\
\text { bem-estar da comunidade }\end{array}$ \\
\hline $\begin{array}{l}\text { Patel, Gauld, } \\
\text { Norris \& Rades, } \\
2010\end{array}$ & $\begin{array}{c}\text { Idosos e } \\
\text { adolescentes (73) }\end{array}$ & $\begin{array}{l}\text { Entrevistas } \\
\text { gravadas }\end{array}$ & Grupo focal & $\begin{array}{c}\text { Medicamentos } \\
\text { gratuitos ou } \\
\text { genéricos } \\
\text { e políticas } \\
\text { públicas }\end{array}$ & $\begin{array}{c}\text { Medicamentos gratuitos ou } \\
\text { genéricos percebidos como } \\
\text { inferiores podem dificultar } \\
\text { implementação de políticas } \\
\text { nacionais para melhorar o } \\
\text { acesso da população aos } \\
\text { medicamentos }\end{array}$ \\
\hline \multicolumn{6}{|c|}{ LILACS - Literatura Latino-Americana e do Caribe em Ciências da Saúde - período de busca não determinado ( $\mathrm{N}=15)$} \\
\hline $\begin{array}{c}\text { Freitas e } \\
\text { Teófilo, } 2010\end{array}$ & $\begin{array}{l}\text { Representantes dos } \\
\text { setores da Saúde, } \\
\text { Assistência Social, } \\
\text { Justiça e idosos (SI) }\end{array}$ & $\begin{array}{c}\text { Avaliação } \\
\text { construtiva }\end{array}$ & Grupo focal & $\begin{array}{c}\text { Violência } \\
\text { contra idosos }\end{array}$ & $\begin{array}{c}\text { Ações limitadas: falta } \\
\text { de transporte e outros } \\
\text { profissionais qualificados; } \\
\text { projeto deve buscar atuação } \\
\text { intersetorial, para atingir } \\
\text { assistência integral; os } \\
\text { idosos consideram válido } \\
\text { e deve ser consolidado no } \\
\text { município. }\end{array}$ \\
\hline $\begin{array}{c}\text { Baldissera e } \\
\text { Bueno, } 2010\end{array}$ & Idosas (6) & $\begin{array}{c}\text { Entrevista } \\
\text { semiestruturada }\end{array}$ & Grupo focal & $\begin{array}{l}\text { Representação } \\
\text { da sexualidade }\end{array}$ & $\begin{array}{l}\text { Concretização de uma } \\
\text { atividade educativa } \\
\text { contextualizada }\end{array}$ \\
\hline $\begin{array}{l}\text { Nieto Murillo, } \\
\text { Cerezo Correa } \\
\text { e Cifuentes } \\
\text { Aguirre, } 2006\end{array}$ & Idosos $(\mathrm{SI})$ & $\begin{array}{c}\text { Entrevista } \\
\text { semiestruturada }\end{array}$ & Grupo focal & $\begin{array}{l}\text { Representação } \\
\text { do processo } \\
\text { saúde-doença }\end{array}$ & $\begin{array}{l}\text { Bem-estar é representado } \\
\text { pela atitude positiva } \\
\text { frente a vida em situações } \\
\text { específicas }\end{array}$ \\
\hline $\begin{array}{l}\text { Gonçalves, } \\
\text { Vahl e Borges, } \\
2001\end{array}$ & Idosos (35) & $\mathrm{SI}$ & Grupo focal & $\begin{array}{l}\text { Idoso contador } \\
\text { de histórias e } \\
\text { envelhecimento } \\
\text { criativamente } \\
\text { bem-sucedido }\end{array}$ & $\begin{array}{l}\text { Contar histórias possibilitou } \\
\text { mudanças significativas: } \\
\text { o papel de contador sem } \\
\text { medo, com descontração e } \\
\text { alegria, aceitando a história } \\
\text { como veículo de interação } \\
\text { intergeracional e de } \\
\text { envelhecimento saudável }\end{array}$ \\
\hline
\end{tabular}

participantes, de seis até 226 idosos informados em oito publicações; porém, mais da metade não apresenta o número de participantes, no total de dez trabalhos.

Quanto aos temas abordados nas investigações, nota-se uma grande variabilidade, pois há preocupações que se repetem apenas quanto à doença de Alzheimer, em dois trabalhos conduzidos no exterior (Hutchings et al., 2010; Frank et al., 2010), e quanto à violência contra idosos, sendo um no Brasil (Freitas \& Teófilo, 2010) e o outro no Canadá (Walsh et al., 2011). Observa-se que as publicações capturadas na base LILACS não trazem nenhum periódico dedicado especificamente à questão do envelhecimento, e os periódicos da área da enfermagem são responsáveis pela metade dos 
trabalhos, enquanto que, dentre os periódicos internacionais que apresentaram artigos sobre o tema em tela, oito são, de algum modo, direcionados às questões relativas aos idosos.

A temática das pesquisas foi muito variada, com estudos sobre escolha do sexo do filho e o desenho de robôs nos quais foi utilizada a técnica focal para perguntar a um grupo de idosos a sua opinião sobre a política de escolha e o uso de métodos de detecção do sexo do filho, como a política do filho único na China (Bhagat et al., 2012) e noutro se investigou a percepção de um grupo de idosos sobre os desenhos de robôs que, no futuro, podem ajudá-los nas atividades diárias (Wu, Fassert \& Rigaud, 2012). Entretanto, na maioria dos trabalhos capturados, foram discutidos temas relacionados mais diretamente aos idosos e ao processo de envelhecimento, tais como as pesquisas estrangeiras de Moreau et al. (2012), Conde (2012), MacKinlay e Dundon (2012) e Zhan et al. (2011) e os trabalhos nacionais de Baldissera e Bueno (2010) e Nieto Murillo, Cerezo Correa e Cifuentes Aguirre(2006), que trataram sobre representação de saúde, doença e sexualidade, lazer, religiosidade, bem-estar e instituição de longa permanência - ILP.

As conclusões dos artigos, na sua maioria, apresentaram de forma positiva os resultados das intervenções realizadas com idosos utilizando grupo focal, desde o levantamento de informações, percepções, avaliações de robôs para assistência de idosos até a violência urbana descrita pelos idosos, como a topografia irregular e a falta de transporte destacadas nos trabalhos de Fadda et al. (2010) e Freitas e Teófilo (2010). As publicações revelaram que o uso da técnica de grupo focal possibilitou a concretização de pesquisas com idosos que favoreceram desde a abordagem inicial sobre uma determinada temática como a escolha de lazer até o detalhamento de informações sobre os espaços urbanos, instituições de longa permanência de idosos, determinantes sociais de saúde, bem-estar e envelhecimento saudável. Observa-se que o grupo focal é um procedimento muito profícuo para a realização de pesquisa com idosos, com as mais diversas temáticas e profundidade.

A despeito do pequeno número de trabalhos publicados sobre grupos focais com idosos no país, e o destaque dos periódicos da área da enfermagem em divulgá-los, a extensão dos temas tratados nas publicações estrangeiras oferece um amplo campo a ser explorado pelos pesquisadores do ambiente acadêmico nacional e pelos profissionais que se encontram envolvidos com as questões do envelhecimento, destacando-se investigações como as de Ellis et al. (2011) e de Fadda et al. (2010), pelo fato de trazerem preocupações que são presentes no ambiente da saúde e de mobilidade, assuntos atuais no Brasil. Por outro lado, além das pesquisas, algumas experiências de intervenção junto à população idosa têm sido conduzidas em nosso meio e podem se tornar referência para trabalhos de outros profissionais.

Relata-se, a seguir, o trabalho de psicólogos na condução de experiências grupais envolvendo a terceira-idade, as quais revelam resultados bastante profícuos para a melhoria da qualidade de vida e bem-estar de idosos. Tais intervenções envolvem diversas técnicas, mas têm, como similaridade, o propósito da promoção de saúde psicológica e a população à qual se voltaram.

\section{Grupo de idosos: uma intervenção psicológica}

A se considerar o baixo número de publicações nacionais de pesquisa sobre o tema intervenção psicológica com idosos, Vitorino (2012) realizou um trabalho com o principal objetivo de avaliar os efeitos de uma intervenção sobre a qualidade de vida de um grupo de idosos atendidos num programa de educação formal em relação à ansiedade, depressão, satisfação com a vida e qualidade de vida percebidas antes e após o processo grupal.

Os participantes dessa pesquisa foram 11 idosos frequentadores de um programa de educação física voltado ao desenvolvimento da autonomia, cuja caracterização revelou um perfil semelhante a diversos trabalhos publicados sobre idosos, tais como: maioria do sexo feminino, com idade média de 66 anos, viúvas, morando com outras pessoas (cônjuge ou filhos), com renda em torno de três salários mínimos. O perfil encontrado por Vitorino (2012) é análogo ao estudo realizado por 
Oliveira, Duarte, Lebrão \& Laurenti(2007), que encontraram dados parecidos em seus estudos, no município de São Paulo, no Estudo SABE (Saúde e Bem-estar e Envelhecimento) e Costa, Ceolim e Neri (2011), que no projeto FIBRA (Fragilidade em Idosos Brasileiros), estudo multicêntrico sobre a fragilidade dos idosos na população brasileira, levantaram características semelhantes.

Vitorino (2012) aplicou quatro instrumentos para medir a ansiedade, a depressão, a qualidade de vida e a satisfação sob o mesmo aspecto, antes e após a intervenção psicológica, tendo avaliado que os efeitos de uma intervenção em grupo, de duração breve (12 sessões, semanais, de uma hora e meia), foram qualitativamente positivos sobre a qualidade de vida dos idosos atendidos, pois ficou claro que todos os participantes conseguiram ter um olhar diferente para os diversos aspectos de sua existência, fato este que não lhes tinha ocorrido antes, favorecendo sua vivência emocional. Quanto à análise, antes e após a intervenção, foi possível verificar que, no grupo, os níveis de ansiedade e de satisfação, praticamente, mantiveram-se os mesmos entre a pré e pós-intervenção. Já os níveis de depressão e a qualidade de vida tiveram ligeira melhora, apresentando resultados estatisticamente significantes nas facetas presente, passado e futuro, participação social e intimidade do WHOQOL-OLD. Na correlação entre os níveis de ansiedade e depressão com a satisfação e a qualidade de vida, antes e após a intervenção, foi verificada, pré-intervenção, forte correlação (com diferença estatisticamente significante) entre ansiedade e depressão, ansiedade e qualidade de vida, e também relacionada à satisfação sob esse aspecto. Na avaliação pós-intervenção, exceto a correlação entre satisfação e qualidade de vida, a correlação estatisticamente significante se manteve entre ansiedade e depressão e ansiedade e qualidade de vida.

É importante frisar que a pesquisa apresenta limitações devido à quantidade de idosos participantes da intervenção $(n=11)$, o que impede a generalização dos dados. Além disso, a complexidade da temática e das variáveis envolvidas não permitiu afirmar com precisão a eficácia da intervenção psicológica na qualidade de vida dos idosos, porém os resultados revelam, principalmente os qualitativos, que os encontros foram positivos, porque permitiram uma maior reflexão sobre aspectos da vida dos participantes (Vitorino, 2012). Vale destacar os comentários de alguns participantes (nomes fictícios) na entrevista devolutiva, que revelaram os benefícios psicológicos da intervenção, como a fala de Guiomar (68 anos): "Apesar de eu gostar muito de ler, faço luta, tae-kwon-do... me senti muito bem... porque você faz o grupo, né?! Tem gente que tem problema pior que o seu", ou nas palavras de Helena (66 anos): "Passei muito tempo remoendo situações do passado, agora decidi olhar para frente e ser feliz, vou viajar com meu marido, somos só nós dois mesmo, para quê regular nossa vida por sofrimentos desnecessários?" (SIC). São tais relatos que corroboram a conjectura de que a vivência em grupo lhes trouxe novas formas de ver a própria vida. Por último, nas palavras de Rosa (66 anos): "Eu tava muito deprimida, quando me falaram do grupo eu vim sem acreditar muito, mas está sendo maravilhoso" (SIC) (Vitorino, 2012, p. 44 e 47).

Essa capacidade que o grupo mostrou de aprender e empregar os conhecimentos em prol de seu bem-estar está de acordo com o que Neri, Yassuda e Cachione (2004) chamaram de velhice bem-sucedida, já que esses participantes estão usando recursos internos próprios para conduzir sua vivência rumo à satisfação plena. Neri (2007, p. 14-15) apresenta um modelo psicológico de bem-estar subjetivo na velhice no qual destaca que "a relação entre os riscos associados ao envelhecimento e o bem-estar subjetivo é mediada pelos mecanismos de autorregulação do self e pelo senso de ajustamento psicológico, em interação com os recursos sociais que os idosos dispõe". O estudo exploratório de Lima e Coelho (2011) utilizou o paradigma do lifespam para analisar a história de vida de oito idosos e investigar as estratégias de seleção, otimização e compensação (SOC) que utilizaram para ter uma velhice bem-sucedida, assim como analisaram as contribuições da geratividade e da participação social no processo de envelhecimento. As autoras apresentam parte dos relatos dos oitos idosos, destacando que os que obtiveram mais sucesso no envelhecer foram os que tiveram um ambiente acolhedor que permitiu o desenvolvimento de estratégias 
de seleção, otimização e compensação, tais como: aumentar os ganhos e diminuir as perdas com acompanhamento médico, de fisioterapia ou de outro profissional da saúde para promover e manter a saúde para superar as debilidades físicas decorrentes da idade.

Os relatos dos 11 participantes da pesquisa de Vitorino (2012) contemplam o modelo psicológico de envelhecimento saudável, na medida em que esses revelaram nas reuniões do grupo de intervenção psicológica não apenas a promoção do bem-estar social e psicológico, mas a análise sobre a própria vida e o seu desenvolvimento humano dentro do processo de envelhecimento. Essa análise sobre o envelhecer e o seu significado permitiu que os idosos avaliassem os riscos e as suas condições físicas, psicológicas e sociais no sentido de procurar viver essa etapa do desenvolvimento humano, denominada velhice, da melhor forma possível, com o objetivo de sentir satisfação com a vida e bem-estar.

Portanto, embora não tenha sido objetivo do trabalho de Vitorino (2012), é claro no relato dos 11 participantes o uso de estratégias de seleção, otimização e compensação, conforme proposto no paradigma do lifespam utilizado na pesquisa de Lima e Coelho (2011), que analisou a história e vida de oito pessoas com mais de 60 anos. De forma semelhante, pode-se afirmar que os idosos da pesquisa de Vitorino (2012) utilizaram e desenvolveram estratégias durante os três meses de intervenção psicológica, pois ao analisar os relatos realizados por eles nos 12 encontros é notório o uso de recursos internos e de autorregulação do self para o enfrentamento de situações características do envelhecimento, como a perda de entes queridos e a compreensão da própria finitude. No grupo de intervenção psicológica, principalmente as mulheres idosas expressavam, de forma clara, os mecanismos que utilizaram, por exemplo, para superar a perda do cônjuge e a aproximação da própria finitude, tais como: não se prender ao passado, viver o presente e aproveitar a vida, seja viajando ou executando o que gosta ou faça com que se sinta realizada.

Os idosos procuram viver bem, de forma que sintam satisfação com a própria vida para que possam ter um envelhecimento bem-sucedido e, consequentemente, saudável e com qualidade de vida. Para tanto, precisam utilizar e aprender novas estratégias de seleção, otimização e compensação para viverem de forma plena a velhice. A técnica de grupo focal utilizada na intervenção psicológica estimulou o desenvolvimento dessas estratégias, pois os assuntos discutidos nos encontros do grupo de idosos permitiram uma melhor compreensão dos diversos aspectos envolvidos no processo de envelhecimento e, em decorrência, na melhoria da qualidade de vida como, por exemplo, a própria participação no grupo e o contato e convívio com outras pessoas, inclusive da universidade (Vitorino, 2012).

Portanto, a vivência em intervenções de grupo e em outros espaços socioculturais é importante para manter e propiciar bem-estar aos idosos. A experiência relatada no artigo de Elias, Pizzeti e Barreto (2013) sobre oficina de passeio com o mesmo tipo de população no espaço urbano revela a importância da ressignificação da cidade e seus espaços públicos, tais como: bosque, lago, biblioteca municipal, aeroporto, entre outros espaços definidos pelo grupo de oito participantes que fizeram parte da intervenção. O resultado da intervenção foi considerado positivo, pois os idosos superaram desde as suas fragilidades físicas até as suas habilidades e competências cognitivas, ou seja, conseguiram percorrer os espaços urbanos, mesmo caminhando com dificuldade ou com a ajuda de uma bengala, e, consequentemente, ressignificaram o espaço urbano e a própria vida.

Tendo em vista as diferenças socioculturais e de idade, a pesquisa de Llobet, Ávila, Farras \& Canut(2011) com uma amostra de idosos octogenários espanhóis, de modo geral, constatou que o conceito de satisfação com a vida leva em consideração os mesmos itens que se destacaram significativamente no estudo de Vitorino (2012) com idosos brasileiros, que são: presente, passado e futuro, seu papel enquanto ser histórico e temporal; participação social como autonomia, independência e adaptação social, e intimidade correspondendo ao companheirismo. Os estudos de Llobet et al. (2011) destacaram que estar satisfeito com a vida não dependia exclusivamente de se ter uma boa qualidade de vida. 
O método de trabalho psicológico de Vitorino (2012) foi o de grupo focal, que ocorre sempre com a delimitação, pelo grupo, de um foco (tema central) que norteia as discussões e que tem relevância para a situação emocional apresentada pelo grupo com vistas a colaborar para a qualidade de vida dos participantes. Nesse modelo de trabalho o psicólogo pesquisador atuou como um orientador das discussões, que deveriam resultar na geração de um novo saber acerca daquele tema que tinha relevância para os participantes. Essas orientações ocorreram conforme andamento dos encontros, pois foram pautadas em temas trazidos pelo grupo, sempre focalizando as questões de interesse dos participantes. Cada encontro teve uma mesma sequência: abertura, discussão, síntese da discussão e fechamento. Foram realizadas atividades lúdicas que contribuíram para a compreensão de aspectos relacionados ao tema discutido pelos idosos, sendo utilizados recursos como: música, textos, dobradura, fotos, lembranças, entre outros. Destaca-se que, a despeito de ser, em sua formulação inicial, um grupo focal, o trabalho envolveu também o aspecto interventivo, na medida em que a expressão de suas preocupações, anseios e a geração do novo saber permitiram alterações nas percepções dos participantes.

\section{Pesquisa e intervenção dirigida ao idoso: a utilização do método clínico}

Em estudos anteriores nota-se que o número de trabalhos com idosos que visa o desenvolvimento de pesquisas com a utilização de intervenção psicológica tem aumentado progressivamente (Gil, 2005, 2010). Nessa perspectiva, diversos estudos têm utilizado o que Turato (2004) denominou de metodologia clínico-qualitativa. Esse tipo de abordagem é fundamentada na observação qualitativa dos fenômenos estudados, em que são valorizadas as angústias e ansiedades existenciais dos participantes do estudo. Nesse sentido, o pesquisador deve valorizar as trocas afetivas na escuta da fala do sujeito e também nos aspectos globais da linguagem corporal e do contato terapêutico. É considerada também como pilar dessa metodologia clínico-qualitativa a compreensão do ser humano pautada nos referenciais teóricos da psicanálise.
Sob a óptica psicanalítica, em uma perspectiva histórica, observamos que as primeiras questões relativas a intervenções psicoterapêuticas dirigidas ao idoso surgiram como tema no início do século XX, introduzidas por Freud (1905/1990), que acreditava na impossibilidade de ser aplicado às pessoas mais velhas o método psicanalítico que criara. Um pouco mais tarde Karl Abraham (1970), ao rever essa ideia, destacou que, no tratamento de pessoas idosas, deveria ser considerada a singularidade dos processos mentais de cada indivíduo e não somente o fator idade, antes de se decidir sobre a possibilidade ou não de ser aplicado o método psicanalítico. Ferenczi (1993) abordou as psiconeuroses do envelhecimento embasado no conceito freudiano do narcisismo, e suas observações surgiram a partir do tratamento de pacientes que não conseguiam, no processo de envelhecimento, modificar a distribuição da libido, ou mesmo adaptá-la frente a essa nova condição, ocorrendo um processo de regressão narcísica.

Nas últimas décadas tornou-se incontestável a ideia da eficácia e indicação da psicoterapia na velhice frente à demanda por cuidados no campo da saúde mental. Quando se pensa em uma clínica que se propõe a atender pessoas na velhice, entrar em contato com o sofrimento psíquico causado, em muitos casos pela exclusão que se dá não só no âmbito social, mas também no familiar, constitui-se um verdadeiro desafio. Além disso, lidar com perdas e limitações físicas, com o medo da proximidade da morte, e o temor, este mais contemporâneo que todos talvez, de ser acometido pelo mal de Alzheimer e tantos outros, são fatores que geram intensas angústias (Chapot, 2009; Gil, 2010).

Nesse sentido, autores como Salvarezza (2005) destacam que a indicação da psicoterapia para o idoso faz parte de um tripé em que estão incluídas a psicofarmacologia, quando necessária, e a psicoterapia de apoio com os familiares. A eficácia terapêutica, então, será relativa aos recursos disponíveis utilizados. A ênfase é dada na importância de uma boa formação do psicanalista, considerando sua formação técnica especializada e análise pessoal. Para o autor, ainda, a maior parte das dúvidas quanto à aplicabilidade da psicanálise da velhice diz respeito, muitas vezes, a aspectos 
da técnica. Desse modo, é comum a aceitação do método psicanalítico pelos próprios psicanalistas, desde que haja modificações (sobre a duração do tratamento, duração das sessões, profundidade das interpretações, etc.). No entanto, o uso da técnica psicanalítica pode ser empregado em qualquer idade e as resistências dos psicanalistas são atribuídas aos preconceitos profundamente arraigados sobre a velhice e, principalmente, aos aspectos contratransferenciais (Salvarezza, 2005).

A visão que considera o tratamento psicanalítico um encontro de inconscientes, e que, portanto, independe tanto da idade do paciente quanto do psicanalista, embasa o pensamento de Messy (1999), autor francês que propõe uma abordagem psicanalítica da velhice. Para o autor, o que está em jogo são os desejos e, por isso, a idade não é um fator que interfere na psique.

Para Chapot (2009), o trabalho psicoterapêutico com idosos, bem como sua inserção em uma rede social, permite-lhes enxergar a possibilidade de experimentar outras maneiras de envelhecer. É possível, assim, dar novos significados a essa fase de vida, resgatando potencialidades, independentemente da idade cronológica. Em seu estudo, ele relata um caso clínico de uma senhora de 68 anos. É descrito um processo, em que a analista, levando em conta a singularidade da paciente, trabalha nas sessões com o foco no relato de sonhos que ela lhe traz quase que semanalmente. É a partir desses relatos que são feitas associações com as várias fases de sua vida. Com o passar do tempo, foi observado que aquela senhora pôde, por meio desse processo, elaborar os conteúdos psíquicos, possibilitando assim alívio do sofrimento e maior integração de self.

Nesse contexto, observa-se que, a despeito da necessidade e da demanda causada pelo sofrimento psíquico, há dificuldade do idoso não só em buscar por psicoterapia, mas também em aderir ao tratamento. Em muitos casos, pode-se atribuir essa questão a dificuldades de adaptação a enquadres mais tradicionais que se traduzem em psicoterapias individuais e de longa duração, nem sempre eficazes, mesmo porque, não raro, o idoso apresenta sérias resistências a procurar ajuda psicológica, por vezes em consequência do próprio preconceito ou também, em muitos casos, por dificuldades econômicas (Gil, 2005).

Nos últimos anos têm sido realizadas pesquisas com o método clínico fundamentadas na observação qualitativa dos fenômenos estudados que propõe intervenções psicoterapêuticas dirigidas ao idoso, tanto individuais como em grupo. Nesse contexto, Schwarz (2009) sugere uma forma de enquadre por meio da psicoterapia grupal breve dirigida aos idosos. A partir do aporte teórico da psicologia analítica, a autora trabalhou com um grupo composto por sete participantes. Foi utilizado o método de Rorschach como teste e reteste para avaliar a evolução de cada pessoa e a do próprio grupo. O foco delimitado foi autoestima e, durante as sessões, foram usados recursos expressivos (desenhos), material onírico e relaxamento. Como conclusões, puderam ser apontados benefícios decorrentes do processo psicoterápico, tais como: maior controle da ansiedade; redução do nível de crítica, do nível de ansiedade e de medos associados às relações interpessoais; não reaparecimento de conteúdos relacionados à impulsividade e ao descontrole; controle emocional mais eficaz e afetividade mais viva, com mais abertura para o contato com o outro.

A pesquisa com enquadres clínicos que utilizam materialidades mediadoras, como elementos de intervenção psicanalítica dirigida ao idoso, inspirada na psicanálise winiccottiana (Gil, Aiello-Vaisberg \& Tardivo, 2009), tem resultado em estudos como o desenvolvido com um paciente de 60 anos, com diagnóstico de transtorno depressivo grave, várias tentativas de suicídio e sucessivas internações psiquiátricas. Nesse tipo de enquadre foram empregados, durante o atendimento psicoterápico, desenho e pintura, com o objetivo de favorecer a comunicação emocional, e também a técnica projetiva TAT, cujas pranchas foram utilizadas não só como elemento diagnóstico, mas também como mediador no contato terapêutico. Após o período de um ano, com atendimento semanal, foi observado que o paciente estava mais integrado, apresentando melhora significativa dos sintomas depressivos, e capaz de romper o ciclo das várias internações psiquiátricas em um curto espaço de tempo. Conclui-se a partir do estudo que a utilização da materialidade mediadora favoreceu a melhora do paciente, 
facilitando a expressão de aspectos emocionais e elaboração dos conteúdos psíquicos (Barros, Gil \& Tardivo, 2010).

Manna e Aiello-Vaisberg (2006, p. 114) relataram o atendimento grupal de idosos por meio da Oficina Psicoterapêutica de Tapeçaria e Outros Bordados, realizado no contexto da Ser e Fazer - Oficinas Psicoterapêuticas de Criação. Trata-se de um enquadre clínico baseado na psicanálise winicottiana e que trabalha com materialidades mediadoras (no caso, lã, talagarça e agulhas), facilitadoras da expressão emocional dos participantes. Segundo as autoras, trata-se de uma clínica que busca a superação da dissociação de aspectos do "self e que oferece cuidados favorecedores da continuidade do ser e do compartilhamento de experiências".

Ainda como exemplo de pesquisa que tem sua fundamentação baseada no método clínico-qualitativo, a título de ilustração, apresentamos um modelo de intervenção psicológica grupal dirigido a idosos denominado Oficina Psicoterapêutica de Cartas, Fotografias e Lembranças. As Oficinas Psicoterapêuticas têm sua fundamentação baseada em um modo de intervenção psicanalítica que utiliza materialidades mediadoras à luz da teoria desenvolvida por D. W. Winnicott e têm se caracterizado por representar um espaço que privilegia tanto o atendimento à comunidade quanto o desenvolvimento da pesquisa clínica. Essa forma de enquadre, a partir de diferentes tipos de materialidades mediadoras, visa oferecer um ambiente terapêutico que possa favorecer o crescimento emocional e o desenvolvimento das potencialidades de cada indivíduo, criando condição de sustentação emocional e recuperação do gesto criativo, podendo assim gerar mudanças (Gil, 2010).

A apresentação da materialidade mediadora em cada oficina é vista como um elemento facilitador do brincar, podendo ser considerado um paradigma do Jogo do Rabisco proposto por Winnicott (1994). A materialidade que é usada como mediação na comunicação entre o terapeuta e o paciente tem a função de gerar condições para a expressão do gesto espontâneo, fazendo com que o paciente saia de um estado de passividade e possa agir sobre o mundo, recuperando assim a possibilidade de existir de modo mais criativo.

Nesse tipo de enquadre se destaca o caráter não interpretativo em que o terapeuta não segue o modelo de "saber sobre o outro", decifrando o que ficou inconscientemente recalcado, mas parte do princípio de que a possibilidade da experiência do encontro inter-humano se fará acompanhar naturalmente da articulação simbólica (Aiello-Vaisberg \& Machado, 2003). Assim, nessa proposta de enquadre diferenciado, segundo Aiello-Vaisberg (2006, p. 26): “A intervenção fundamental neste caso será o manejo ou holding, mediante o qual se exerce um cuidado à continuidade de ser, que favorece movimentos no sentido da integração pessoal que se encontra no gesto criador".

No enquadre clínico proposto da Oficina Psicoterapêutica de Cartas, Fotografias e Lembranças, os idosos recebem o convite para que tragam cartas, fotografias, objetos ou ainda lembranças de qualquer natureza. Os encontros são semanais e têm uma hora e meia de duração. Sobre um cavalete ou mesa, apoia um quadro magnético branco que, auxiliado por ímãs, recebe e sustenta os diferentes materiais trazidos em cada sessão. O beiral do cavalete ou a superfície da mesa fornecem ainda a base para que sejam apoiados objetos que não puderem ser afixados no quadro. Após afixar o material trazido para a sessão, os participantes posicionam-se ao redor do quadro, quando, então, é aberto um espaço para falarem sobre as recordações e experiências que essas materialidades suscitam, bem como sobre tantos outros acontecimentos que fazem parte da vida de cada um. Desse modo, no espaço potencial criado, no conjunto, ocorre a manifestação de uma expressão coletiva, mas que conserva também o particular e singular de cada indivíduo.

Ao final da sessão, o quadro magnético é fotografado e a fotografia compõe um álbum que registra a produção do grupo. Durante as sessões o álbum permanece no local e fica disponível aos participantes. Ao serem fotografados novamente pela terapeuta, as fotografias e objetos instituem uma nova lembrança, contribuindo assim para o registro 
daquele momento e também, de modo mais amplo, para a construção da história do grupo.

Nessa oficina, portanto, os participantes trazem lembranças de um passado mais recente ou distante, que são contadas ao grupo. Muitas vezes essas lembranças são mediadas por materialidades concretas: cartas, fotografias e objetos variados, a partir dos quais surgem as recordações. Segundo Machado, Aiello-Vaisberg, Gil \& Tardivo(2003, p. 68), os objetos trazidos às sessões podem ser designados como objetos dramáticos, pois:

[...] provêm da história de vida de cada um e têm o poder de constelar, no aqui e agora, uma experiência emocional significativa do passado [...] esta expressão enfatiza o enraizamento do objeto na trajetória do viver pessoal, bem como o fato de virem entretecidos com narrativas de histórias de vida.

Por meio desses objetos dramáticos, acontece um mundo transicional, onde o passado (que pode ser recente ou mais remoto) se presentifica. Segundo a observação de Machado et al. (2003), o que viabiliza a experiência vivida voltar a vida no presente é o fato desta poder ser compartilhada, do ponto de vista emocional, pelo grupo. Desse modo, ao dar um novo sentido às experiências passadas, favorecido por trocas afetivas que ocorreram no presente, é possível também projetar um futuro de novas perspectivas.

A integração entre a materialidade trazida pelos participantes e a narrativa referente às recordações despertadas dá uma dimensão existencial ao encontro terapêutico, ou em outras palavras, o gesto espontâneo pode ser assim integrado à narrativa. Vale dizer que gesto espontâneo é um termo criado por Winnicott (1983) para designar uma das formas de expressão do self verdadeiro. Nesse contexto, deve ser levado em conta o holding fornecido ao grupo pela presença e disponibilidade emocional da terapeuta que, à medida que acolhe os objetos dramáticos, favorece um campo propício para a troca e elaboração de experiências emocionais (Machado et al., 2003).

Ao longo dos últimos anos têm sido realizadas pesquisas com o enquadre clínico proposto na
Oficina Psicoterapêutica de Cartas, Fotografias e Lembranças, em diferentes contextos. Nesse sentido, foi realizada uma intervenção dirigida a um grupo formado por cinco homens com idade entre 60 e 73 anos que apresentavam sintomas de depressão. Esse grupo mantinha, na época do estudo, a mesma configuração há cerca de um ano. Na forma de vinhetas clínicas, foi discutido o processo psicoterapêutico de três participantes, a partir do convite para que os pacientes trouxessem cartas, fotografias ou objetos às sessões (Gilet al., 2009).

Um dos participantes, com 70 anos de idade, que apresentava grande sofrimento pela morte abrupta do filho, pôde, após seis meses de participação, começar a trazer às sessões fotografias do filho e, com o apoio do grupo, passou a montar, durante as sessões, um álbum referente a várias fases da vida do filho. Do mesmo modo, outro participante de 60 anos, que se angustiava muito com diversas mudanças em sua vida (a morte da mãe e a mudança de casa), pôde elaborar melhor esses conteúdos a partir de fotografias que retratavam sua casa antiga e também a nova moradia, além de cartas que havia recebido de sua mãe. Por fim, o terceiro participante, também com 60 anos, bastante deprimido após a amputação de uma perna devido à diabetes, aceitou o convite de outro integrante para participar do coral, sendo marcante a sessão na qual ele traz um convite para que o grupo fosse assistir a ele na apresentação no final do ano (Gil et al., 2009).

Esses estudos resultaram na realização de uma pesquisa (Gil, 2010) que objetivou apresentar a oficina em questão como proposta de método psicoterapêutico dirigido a idosos em um enquadre grupal e verificar seu alcance e seus benefícios para esse grupo. A pesquisa foi desenvolvida com o método clínico baseado na teoria psicanalítica e, em especial, na leitura dos conceitos winnicottianos.

A proposta terapêutica, descrita no trabalho, foi desenvolvida a partir dos temas de recordação e transicionalidade, cujos efeitos foram avaliados antes e depois da intervenção. Participaram do estudo seis idosos de ambos os sexos - um homem e cinco mulheres - com idade entre 65 e 79 anos, que frequentavam um grupo de terceira idade. Para compreensão diagnóstica, foram 
realizadas duas entrevistas iniciais e aplicados os instrumentos WHOQOL-BREF (Inventário de Qualidade de Vida - forma breve), o BDI - Inventário Beck de Depressão e o Teste de Apercepção Temática para Idosos - SAT. Ao final do processo foi realizada uma nova entrevista individual com os participantes, quando foram reaplicados os mesmos instrumentos da entrevista inicial, com exceção do SAT, cuja reaplicação não é recomendada em um período anterior há um ano. O estudo descreve o processo psicoterapêutico desenvolvido, realizado em grupo e de forma breve, durante 16 sessões, e as mudanças que ocorreram na vida dos participantes.

No que diz respeito ao enquadre utilizado na Oficina Psicoterapêutica, observou-se que as fotografias e os objetos facilitaram a recordação das lembranças e a expressão dos aspectos emocionais dos participantes. A partir dos relatos no grupo, notou-se que a materialidade favoreceu uma maneira de recordar que não está somente ligada a perdas, mas que de outro modo permitiu aos participantes o acesso às suas reminiscências (Salvarezza, 2003). Assim, durante o processo da oficina, gradualmente foi se estabelecendo um modo de recordar mais saudável, o que se refletiu no aumento da capacidade de valorizar os ganhos e na reafirmação da autoestima dos participantes.

Em um ambiente terapêutico auxiliado pelo holding (Winnicott, 1983, 2000) do grupo ocorreu um campo propício para o compartilhamento de experiências entre os participantes e a elaboração dos conteúdos psíquicos. As fotografias e os objetos foram considerados em seu caráter transicional, à medida que permitiram estabelecer a ligação entre passado e presente, por meio da recordação das lembranças que despertaram.

Ao final do processo da oficina psicoterapêutica, a observação clínica, os relatos individuais e os resultados descritos pelos instrumentos objetivos que foram utilizados, demonstraram significativa melhora dos sintomas depressivos apresentados inicialmente e, de modo geral, melhora da qualidade de vida de todos os que concluíram a participação no grupo. Entre outros aspectos, pôde-se perceber nos participantes maior abertura para o mundo e mais proximidade de si mesmos e de seus sentimentos.

O enquadre diferenciado, realizado de forma breve e em grupo, a exemplo de outros estudos, se mostrou eficaz, facilitando a aderência dos participantes à proposta terapêutica. $\mathrm{O}$ atendimento realizado com idosos em Oficinas Psicoterapêuticas, ao utilizar materialidades mediadoras facilitadoras da expressão emocional, mostrou-se capaz de atender às necessidades do idoso por cuidados no campo emocional. Como conclusão deste estudo, considerou-se que os objetivos propostos foram contemplados e a proposta de método psicoterapêutico desenvolvida com esse grupo se mostrou eficaz, considerando a compreensão clínica do processo, a reaplicação dos instrumentos e a visão dos próprios participantes.

\section{Considerações finais}

A partir da constatação do pequeno número de pesquisas envolvendo grupos focais publicadas em nossos periódicos e da grande abrangência de temas aos quais se prestam, há um amplo campo para os profissionais das mais diversas áreas de conhecimento para conduzirem estudos envolvendo tal técnica. Ainda, como verificado na literatura científica internacional, os grupos, quer investigativos, quer terapêuticos, com diversos formatos, apresentam-se como profícuos à promoção de saúde e bem-estar à população que envelhece, e que se amplia a cada dia. Além disso, entende-se que a interdisciplinaridade é, seguramente, fundamental para atender, de modo amplo, os idosos de hoje e preparar a sociedade para os que mais virão.

Nesse sentido, são necessários maiores investimentos públicos na formação de recursos humanos, principalmente, de profissionais da saúde e, em específico, da área da Psicologia, para a realização de mais estudos científicos e o desenvolvimento de intervenções psicológicas. Por outro lado, a técnica de grupo focal pode contribuir para prevenir, manter e promover a saúde e bem-estar dos idosos e daqueles que cuidam e convivem com o seu processo de envelhecimento.

O presente artigo tem limitações, pois o objetivo não foi fazer uma revisão sistemática 
ou uma metanálise sobre o tema da pesquisa qualitativa, destacando o grupo focal e as intervenções psicológicas baseadas na metodologia clínico-qualitativa com idosos. A proposta foi fazer uma análise crítica sobre o tema e apresentar aos leitores a importância das pesquisas qualitativas, do método clínico e da utilização da técnica de grupo focal para levantar, focalizar e aprofundar o conhecimento sobre um determinado tema com base nas discussões realizadas pelos grupos de idosos que participam das intervenções organizadas para a coleta de dados. Nota-se que, ainda, há muito trabalho de intervenção psicológica para ser realizado com os idosos utilizando a técnica de grupo focal. 


\section{Ana Lúcia Gatti}

Doutora em Psicologia pela Pontifícia Universidade Católica de Campinas, São Paulo - SP. Brasil. Docente na Universidade São Judas Tadeu, São Paulo - SP. Brasil.

E-mail: algatti@ig.com.br

\section{Carla Witter}

Doutora em Psicologia Escolar e do Desenvolvimento da Universidade de São Paulo, São Paulo - SP. Brasil. Docente da Universidade São Judas Tadeu, São Paulo - SP. Brasil.

E-mail: cwitter12@gmail.com

\section{Claúdia Aranha Gil}

Doutora em Psicologia pela Universidade de São Paulo, São Paulo - SP. Brasil. Docente da Universidade São Judas Tadeu, São Paulo - SP. Brasil. E-mail: claudiaagil@uol.com.br

\section{Sueli dos Santos Vitorino}

Mestre em Ciências do Envelhecimento pela Universidade São Judas Tadeu, São Paulo - SP. Brasil. E-mail: suelidosantos@ig.com.br

Endereço para envio de correspondência:

Universidade São Judas Tadeu. Rua Taquari, 546. Mooca. CEP 03166-000 - São Paulo, SP Brasil.

Recebido 06/12/2013, Aprovado 01/10/2014. 
Abraham, K. (1970). Teoria psicanalítica da libido: sobre o caráter e o desenvolvimento da libido (Christiano Monteiro Oiticica, trad.). Rio de Janeiro: Imago. (Trabalho original publicado em 1927).

Aiello-Vaisberg, T. M. J. (2006). Efeitos clínicos da ArteterapiaWinnicottiana. In T. M. J. AielloVaisberg \& F. F. Ambrosio (Orgs.), Cadernos ser e fazer: imaginários coletivos como mundos transicionais. São Paulo: Instituto de Psicologia da Universidade de São Paulo.

Aiello-Vaisberg, T. M. J., \& Machado, M. C. L. (2003). Sofrimento humano e estudo da "eficácia terapêutica" de enquadres clínicos diferenciados. In T. M. J. Aiello-Vaisberg \& F. F. Ambrosio (Orgs.), Cadernos ser e fazer: imaginários coletivos como mundos transicionais. São Paulo: Instituto de Psicologia da Universidade de São Paulo. Recuperado em http://serefazer.psc.br/wp-content/ uploads/2012/09/christina-tem\%C3\%A1ticopara-caderno-verde-10ago03.pdf

Backes, D. S., Colomé, J. S., Erdmann, R. H., \& Lunardi, V. L. (2011).Grupo focal como técnica de coleta e análise de dados em pesquisas qualitativas. O Mundo da Saúde, 35(4), 438442. Recuperado em http://www.saocamilo-sp. br/pdf/mundo_saude/88/10_GrupoFocal.pdf

Baldissera, V. D. A., \& Bueno, S. M. V. (2010). A representação da sexualidade por idosas e a educação para a saúde. Revista Eletrônica de Enfermagem, 12(4), 622-629..

Barros, M. S. M., Gil, C. A., \& Tardivo, L. S. L. P. C. (2010). Empleo de encuadres diferenciados em el contexto clínico: el uso de materialidades como mediadoras em elprocesoterapêuticode um paciente com depresión grave. In A. Trimboli. Trauma, história y subjetividad.V CongresoArgentino de Salud Mental (pp. 66-67). Buenos Aires: Assoc. Argentina de Profesionales de Salud Mental.

Bhagat, N., Laskar, A. R., \& Sharma, N. (2012). Women's perception about sex selection in an urban slum in Delhi. Journal of Reproductive and Infant Psychology, 30(1), 92-104. http:// dx.doi.org/10.1080/02646838.2011.635297.

Bosi, E. (2003). O tempo vivo da memória: ensaios de psicologia social. São Paulo: Atêlie.

Bruns, M. A. T., \& Abreu, A. S. (1997). O envelhecimento: encantos e desencantos da aposentadoria. Revista da ABOP, 1(1), 5-33. Recuperado em http://pepsic.bvsalud.org/ scielo.php?script $=$ sci arttext $\&$ pid $=$ S1414$88891997000100002 \& \operatorname{lng}=$ pt\&nrm $=$ iso

Carlini-Cotrim, B. (1996). Potencialidades da técnica qualitative grupo focal em investigações sobre abuso de substâncias. Revista de Saúde Pública, 30(3), 285-293. http://dx.doi.org/10.1590/ S0034-89101996000300013. PMid:9110476

Chapot, S. L. (2009). A psicoterapia enlavejez: un caso clínico. In Temas de psicogerontologia: investigación, clínica y recursos terapéuticos. Buenos Aires: Librería Akadia.

Cheng, J. O., Lo, R., Chan, F., \& Woo, J. (2010). A pilot study on the effectiveness of anticipatory grief therapy for elderly facing the end of life. Journal of Palliative Care, 26(4), 261-269. PMid:21268518.

Conde, M. D. (2012). Leisure patterns and needs of the elderly in rural Galicia (Spain). Educational Gerontology, 38(2), 138-145. http://10.1080/03601277. 2010. 515894

Costa, S. V., Ceolim, M. F., \& Neri, A. L. (2011). Problemas de sono e suporte social: estudo multicêntrico Fragilidade em Idosos Brasileiros. Revista Latino-Americana de Enfermagem, 19(4). http://dx.doi.org/10.1590/S010411692011000400010. PMid:21876944

Coutinho, M. C., Diogo, M. F., Joaquim, E. P., \& Borges, R. C. P. (2011). O trabalho de manutenção em uma prefeitura universitária: entre dificuldades e realizações. Psicologia: Ciência e Profissão, 31(1), 96-109. http://dx.doi. org/10.1590/S1414-98932011000100009.

Dawalibi, N. W., Anacleto, G. M. C., Witter, C., Goulart, R. M. M., \& Aquino, R. (2013). Envelhecimento e qualidade de vida: análise da produção científica da SciELO. Estudos de Psicologia, 30(3), 393-403. http://dx.doi. org/10.1590/S0103-166X2013000300009.

Debert, G. G. (1999). A reinvenção da velhice: socialização e processos de reprivatização do envelhecimento. São Paulo: Edusp.

Elias, M. V., Pizzeti, S. A., \& Barreto, D. J. (2013). Idosos em experimentação no circuito urbano: relato de uma experiência. Psicologia: Ciência e Profissão, 33(3), 746-757. http://dx.doi. org/10.1590/S1414-98932013000300017.

Ellis, J. C.; Mullan, J., \& Worsley, T. (2011). Prescription medication hoarding and borrowing or sharing behaviours in older residents in the Illawarra, 
New South Wales, Australia. Australasian Journal on Ageing, 30(3), 119-123</jrn>.

Fadda, G., Cortés, A., Olivi, A., \& Tovar, M. (2010). The perception of the values of urban space by senior citizens of Valparaiso. Journal of Aging Studies, 24(4), 344-357. http://dx.doi. org/10.1016/j.jaging.2010.07.001.

Ferenczi, S. (1993). Psicanálise III. In S. Ferenczi.Obras completas. Para compreender as psiconeuroses do envelhecimento. Alvares Cabral, trad., cap. 17, pp. 145-150). São Paulo: Martins Fontes. (Trabalho original publicado em 1939).

Frank, L., Howard, K., Jones, R., Lacey, L., Leibman, C., Lleo, A., et al (2010).A qualitative assessment of the concept of dependence in Alzheimer's disease. American Journal of Alzheimer's Disease and Other Dementias, 25(3), 239-247. http:// dx.doi.org/10.1177/1533317509356690. PMid:20147602

Freitas, C. A. S., \& Teófilo, T. J. F. (2010). Avaliação construtivista, sob uma abordagem integradora e intersetorial, das ações do Projeto Disque Idoso em Sobral (CE, Brasil). Ciencia \& Saude Coletiva, 15(6), 2825-2833. http://dx.doi. org/10.1590/S1413-81232010000600020. PMid:20922291

Freud, S. (1990). Sobre a psicoterapia. In Edição Standard Brasileira das Obras Completas de Sigmund Freud (3. ed., Vol. 7). Rio de Janeiro: Imago. (Trabalho original publicado em 1905).

Gil, C. A. (2005). Envelhecimento e depressão: da perspectiva psicodiagnóstica ao encontro terapêutico.Dissertação de Mestrado em Psicologia, Instituto de Psicologia da Universidade de São Paulo, São Paulo, SP.

Gil, C. A. (2010). Recordação e transicionalidade: a oficina de cartas, fotografias e lembranças como intervenção psicoterapêutica grupal com idosos. Tese de Doutorado, Instituto de Psicologia da Universidade de São Paulo, São Paulo, SP. Recuperado em www.teses.usp.br/ teses/ disponiveis/47/47133/tde-20012011 .../ gil_do2.pdf

Gil, C. A., Aiello-Vaisberg, T. M. J., \& Tardivo, L. S. L. P. C. A. (2009). Oficina psicoterapêutica de cartas, fotografias e lembranças: uma experiência inclusiva. In D. Hur et al. Ansi do XVIII Congresso Latino Americano FLAPAG e X Simpósio CEFAS: práticas institucionais na América Latina: Casal, Família, Grupo e Comunidade (pp. 285-285). Campinas.
Gonçalves, L. H. T., Vahl, E. A. C., \& Borges, I. C. (2001).O idoso contador de histórias: a autopromoção de um envelhecimento criativamente bem sucedido. Texto \& ContextoEnfermagem, 10(2), 94-115.

Hutchings, D., Vanoli, A., Mckeith, I., Brotherton, S., Mcnamee, P., \& Bond, J. (2010). Cholinesterase inhibitors and Alzheimer's disease: Patient, carer and professional factors influencing the use of drugs for Alzheimer's disease in the United Kingdom. Dementia. The International Journal of Social Research and Practice, 9(3), 427-443. http://dx.doi.org/10.1177/1471301210375340.

Iliffe, S., Kharicha, K., Harari, D., Swift, C., Goodman, C., \&Manthorpe, J. (2010).User involvement in the development of a health promotion technology for older people: findings from the SWISH project. Health \& Social Care in the Community, 18(2), 147-159. http://dx.doi.org/10.1111/j.13652524.2009.00882.x.PMid:19708867

Kernberg, O. F. (2000). Ideologia, conflito e liderança em grupos e organizações. Porto Alegre: Artes Médicas Sul.

Lima, P. M. R., \& Coelho, V. L. D. (2011). A arte de envelhecer: um estudo exploratório sobre a história de vida e o envelhecimento. Psicologia: Ciência e Profissão, 31(1), 4-19. http://dx.doi. org/10.1590/S1414-98932011000100002.

Llobet, M. P., Ávila, N. R., Farras, J. F., \& Canut, M. T. L. (2011). Qualidade de vida, felicidade e satisfação com a vida em anciãos com 75 anos ou mais, atendidos num programa de atenção domiciliária. Rev. Lat. Amer. Enfermagem, 19(3). http://dx.doi.org/10.1590/S010411692011000300004. [online]

Machado, M. C. L., Aiello-Vaisberg, T. M. J., Gil, C. A., \& Tardivo, L. S. L. P. C. (2003). Oficina psicoterapêutica de cartas, fotografias e lembranças: uma experiência dramática. In T. M. J. Aiello-Vaisberg \& F. F. Ambrosio (Orgs.), Cadernos ser e fazer: apresentação e materialidade (pp. 66-81). São Paulo: Instituto de Psicologia da Universidade de São Paulo. Recuperado em http://serefazer.psc.br/wpcontent/uploads/2012/09/OficinadeCartas_ CadernoVerde.pdf

MacKinlay, E., \& Dundon, C. (2012). An exploration of health and religion in elderly people through the lens of scriptural reminiscence. Journal of Religion, Spirituality and Aging, 24(1-2), 42-54. http://dx.doi.org/10.1080/15528030 .2012 .632714 . 
Manna, R. E., \& Aiello-Vaisberg, T. M. J. (2006). Oficina psicoterapêutica de tapeçaria e outros bordados: bordando a vida ponto-a-ponto. In T. M. J. Aiello-Vaisberg \& F. F. Ambrosio(Orgs.), Cadernos ser e fazer: imaginários coletivos como mundo transicionais (pp. 95-116).São Paulo: Instituto de Psicologia da Universidade de São Paulo.

Messy, J. (1999). A pessoa idosa não existe: uma abordagem psicanalítica da velhice. São Paulo: Aleph.

Minayo, M. C. S. (2006).O desafio do conhecimento: pesquisa qualitativa em saúde. 9a edição revista e aprimorada. São Paulo: Hucitec.

Moreau, A., Carol, L., Dedianne, M. C., Dupraz, C., Perdrix, C., Lainé, X., et al. (2012). What perceptions do patients have of decision making (DM)? Toward an integrative patientcentered care model: a qualitative study using focus-group interviews. Patient Education and Counseling, 87(2), 206-211.

Neri, A. L. (2007). Qualidade de vida na velhice e subjetividade. In A. L. Neri (Org.), Qualidade de vida na velhice: enfoque multidisciplinar. Campinas: Alínea.

Neri, A. L., Yassuda, M. S., \& Cachioni, M. (2004). Velhice Bem sucedida: Aspectos afetivos e cognitivos. São Paulo: Papirus.

Nieto Murillo, E., Cerezo Correa, M. P., \& Cifuentes Aguirre, O. L. (2006). Representaciones de la Vejez en Relación con el Procesosaludenfermedad de ungrupo de ancianos: old age representation in relation with the healthdisease process of elderly people. Hacia Promoción de la Salud, (11), 107-118.

Oliveira, S. F. D., Duarte, Y. A. O., Lebrão, M. L., \& Laurenti, R. (2007). Demanda referida e auxílio recebido por idosos com declínio cognitivo no Município de São Paulo. Revista Saúde e Sociedade, 16(1), 81-89.

Oliveira, M., \& Freitas, H. M. R. (1998). Focus Group: pesquisa qualitativa: resgatando a teoria, instrumentalizando o seu planejamento. Revista de Administração, 33(3), 83-91. Recuperado em http://www.rausp.usp.br/busca/artigo. asp?num_artigo $=258$

Osório, L. C. (2003). Entendendo e atendendo sistemas humanos. In W. J. Fernandes, B. Svartman \& B. S. Fernandes. Grupos e configurações vinculares (pp. 57-62). Porto Alegre: Artmed.
Patel, A., Gauld, R., Norris, P., \& Rades, T. (2010). “This body does not want free medicines": South African consumer perceptions of drug quality. Health Policy and Planning, 25(1), 61-69. http://dx.doi.org/10.1093/heapol/czp039. PMid:19726560

Rubio, M. E., Wanderley, K. S., \& Ventura, M. M. (2011).A viuvez: a representação da morte na visão masculina e feminina. Kairós Gerontologia, 14(1), 137-147.

Salmaso, F. V., Vigário, P. S., Mendoça, L. M. C., Madeira, M., Netto, L. V., Guimarães, M. R. M. et al. (2014). Análise de idosos ambulatoriais quanto ao estado nutricional, sarcopenia, função renal e densidade óssea. Arquivos Brasileiros de Endocrinologia e Metabologia, 2014, 58-63. http://dx.doi.org/10.1590/00042730000002580.

Salvarezza, L. (2003). Psicogeriatria teoria y clinica. Buenos Aires: Paidós.

Salvarezza, L. (2005). La vezes: unamiradagerontológica actual. Buenos Aires: Paidós.

Schwarz, L. R. (2009). EnvelheSer: a busca do sentido da vida na terceira idade. Um processo de psicoterapia grupal breve de orientação junguiana. São Paulo: Vetor.

Soares, C. B., Reale, D., \& Brites, C. M. (2000). Uso de grupo focal como instrumento de avaliação de programa educacional em saúde. Revista da Escola de Enfermagem da USP, 34(3), 317-322. http://dx.doi.org/10.1590/S008062342000000300014. [online] PMid:12033140

Turato, E. R. (2004). A questão da complementaridade e das diferenças entre métodos quantitativos e qualitativos de pesquisa: uma discussão epistemológica necessária. In S. Grubits \& J. A. V. Noriega. Método Qualitativo:epistemologia, complementariedades e campos de aplicação. São Paulo: Vetor, p.17-51.

Vitorino, S. S. (2012). Qualidade de vida percebida por idosos de um programa educativo: avaliação de uma intervenção psicológica. Dissertação de Mestrado,Ciências do Envelhecimento, Universidade São Judas Tadeu, São Paulo, SP. Recuperado em http://www.usjt.br/biblioteca/ mono_disser/mono_diss/2012/201.php

Walsh, C. A., Olson, J. L., Ploeg, J., Lohfeld, L., \& MacMillan, H. L. (2011).Elder abuse and oppression: voices of marginalized elders. Journal of Elder Abuse \& Neglect, 23(1), 17-42. 
http://dx.doi.org/10.1080/08946566.2011.5 34705. PMid:21253928

Winnicott, D. W. (1983). Contratransferência. In O ambiente e os processos de maturação (pp.145-151). Porto Alegre: Artes Médicas. (Trabalho original publicado em 1960).

Winnicott, D. W. (1994). O jogo do rabisco (Squiggle Game). In C. Winnicott \& R. Shephard (Orgs.), Explorações psicanalíticas (pp. 230-243). Porto Alegre: Artes Médicas. (Trabalho original publicado em 1968).

Winnicott, D. W. (2000). Objetos Transicionais e fenômenos transicionais. In Da pediatria a psicanálise (pp. 316-331). Rio de Janeiro: Imago. (Trabalho original publicado em 1951).

Witter, C., Buriti, M. A., Silva, G. B., Nogueira, R. S., \& Gama, E. F. (2013). Envelhecimento e dança: análise da produção científica na Biblioteca Virtual de Saúde. Revista Brasileira de Geriatria e Gerontologia, 16(1), 191-199. http://dx.doi. org/10.1590/S1809-98232013000100019.

Witter, G. P. (Org.) (2010).Envelhecimento: referenciais teóricos e pesquisas (2. ed.). Campinas.

Witter, C., \& Buriti, M. A. (Orgs).(2011). Envelhecimento e contingências da vida. Campinas: Alínea.

Wu, Y. H., Fassert, C., \& Rigaud, A. S. (2012). Designing robots for the elderly: appearance issue and beyond. Archives of Gerontology and Geriatrics, 54(1), 121-126. http:// dx.doi.org/10.1016/j.archger.2011.02.003. PMid:21349593

Zhan, H. J., Feng, Z., Chen, Z., \& Feng, X. (2011). The role of the family in institutional long-term care: Cultural management of filial piety in China. International Journal of Social Welfare, 20(Suppl 1), S121-S134.http://dx.doi. org/10.1111/j.1468-2397.2011.00808.x. 